\title{
Análise de referências em teses e dissertações sobre uso de Computação Afetiva na área da educação
}

\author{
João R. U. da Cruz ${ }^{1,2}$, Wellington B. Rodrigues ${ }^{1}$, Carla Rodriguez ${ }^{1}$, Denise Goya ${ }^{1}$ \\ 1 - Pós-graduação em Ciência da Computação, UFABC - Santo André - SP \\ 2 - Universidade Santo Amaro - UNISA - São Paulo - SP \\ \{cruz.joao, wellington.rodrigues, c.rodriguez, \\ denise.goya\} @ufabc.edu.br
}

\begin{abstract}
Resumo. O uso da Computação Afetiva no ambiente computacional para a educação vem sendo objeto de diversas pesquisas na área acadêmica, e especialmente as stricto- sensu. A formação das hipóteses e teorias desses pesquisadores são apresentadas nessas pesquisas por meio das citações de outros autores. Este artigo analisou 21 Teses e Dissertações sobre a Computação Afetiva no ambiente computacional para a educação visando identificar quais são as fontes de citação mais usadas, e como elas influenciaram as pesquisas. Autores como PICARD e EKMAN, e seus trabalhos são as referências que aparecem com maior frequência nas redes de citação utilizadas nessas pesquisas, e influenciaram, principalmente, as definições de Computação Afetiva e Emoções Básicas.
\end{abstract}

\begin{abstract}
The use of Affective Computing in the computational environment for education has been the subject of several researches in the academic area, and especially the stricto-sensu. The formation of the hypotheses and theories of these researchers are presented in these studies through citations by other authors. This article analyzed 21 Theses and Dissertations on Affective Computing in the computational environment for education in order to know which are the most used sources of citation, and how they influenced research. Authors such as PICARD and EKMAN, and their works are the references that appear most frequently in the citation networks used in these researches, and influenced mainly with the definitions of Affective Computing and Basic Emotions.
\end{abstract}

\section{Introdução}

"Computação que se relaciona com, surge, ou deliberadamente influencia emoções." Assim PICARD (1997) definiu a Computação Afetiva dando início a uma nova abordagem, que considera o computador, na área da informática na educação, como objeto de estímulo para o desenvolvimento cognitivo através das emoções. Essa nova abordagem foi pesquisada por diversas áreas do conhecimento, como psicologia, neurociência, linguística entre outras [PAIM(2016), SANTANA(2017)]. Na ciência da computação, o desenvolvimento das pesquisas sobre essa nova abordagem dentro do ambiente escolar fez-se de duas formas: as pesquisas sobre a interação homemcomputador [ZUO et. al. (1998), NIJHOLT \& POPPE(2008)], e na simulação das emoções [GONZALEZ \& WOODS(2012), TAN et. al.(2012)].

A associação da computação ao processo ensino-aprendizagem estimulou pesquisas sobre o uso da Computação Afetiva associada ao ensino a distância, à gamificação, e outras estratégias de ensino-aprendizagem, que permitiram o desenvolvimento de diversas teorias sobre o aprendizado afetivo [FULLER, VICIAN \& BROW(2016), GONÇALVES(2016), STIPEK(2002)]. Os laboratórios computacionais dentro das 
instituições de ensino, o uso de dispositivos móveis em sala de aula, e até o fácil acesso a câmeras e sensores, contribuíram para que professores e pesquisadores pudessem registrar, e associar as reações afetivas durante o processo de ensino-aprendizagem [GOTTARDO(2018), SANTANA(2016)]. Essas teorias estimularam também o surgimento de pesquisas stricto-sensu sobre o uso da Computação Afetiva na educação.

Este artigo visa analisar de forma sistemática as fontes de citação das pesquisas[MORAES, FURTADO \& TOMAÉL(2015)] stricto-sensu sobre o uso da Computação Afetiva em ambientes computacionais de ensino-aprendizagem, tendo como objetivo identificar quais são as principais referências utilizadas nestas pesquisas, quais seus principais autores de referência, e como esses trabalhos influenciaram essas pesquisas.

Na próxima seção deste artigo apresentamos a metodologia utilizada para a busca das teses e dissertações, assim como descrevemos a técnica para coleta e análise das referências bibliográficas. Na terceira seção, é possível encontrar a análise dos dados e posteriormente, na última seção, apresentamos as considerações finais sobre a pesquisa.

\section{Metodologia}

A análise da rede de citações permite a identificação de quais são os trabalhos mais influentes, e quem são os respectivos autores [MORAES, FURTADO \& TOMAÉL(2015)] evidenciando quais foram as fontes fornecedoras de maior quantidade de insumos intelectuais para a formação das teorias apresentadas pelo pesquisador. [HORTON(2008)].

Nesse contexto, os objetivos deste artigo são: (i) Identificar quantos, e quais foram as pesquisas stricto-sensu sobre Computação Afetiva em ambientes computacionais, voltados à educação, entre 2013 e 2018. (ii) Identificar quantos e quais os trabalhos mais citados nessas pesquisas. (iii) Identificar quais são os autores com maior influência sobre essas pesquisas.

Para atingir os objetivos estabelecidos optamos por dividir nosso protocolo de ações em três partes: 1. Coleta de Dados; 2. Pré-processamento; e 3. Criação da rede de Citações.

\subsection{Coleta de dados}

Para realizar a busca de Teses e Dissertações publicadas no Brasil sobre Computação Afetiva utilizamos o Catálogo de Teses e Dissertações (CTD) da Fundação Coordenação de Aperfeiçoamento Pessoal de Nível Superior (CAPES) como ferramenta de busca. Para que o documento fosse aceito nesta pesquisa, ele deveria constar no CTD, e estar disponível para download pela própria ferramenta, ou em repositório de acesso público da Universidade no qual o documento foi defendido.

Para realização da busca utilizamos a string "computação afetiva" or "affective computer" or "emoções" or "emotions" or "sentimentos" or "feelings". Também aplicamos filtros de refinamento da busca na própria ferramenta no CTD, com os seguintes critérios: Área de Conhecimento: Ciências da Computação ou Sistemas de Informação, Área de Concentração: Ciências da Computação e Anos: 2013, 2014, 2015, 2016, 2017 e 2018. Como resultado preliminar retornaram 757 Dissertações e 176 Teses, totalizando 933 trabalhos.

Como segunda etapa da seleção dos trabalhos, realizamos uma busca manual nas teses e dissertações pelos termos, "AFETIVIDADE”, "EMOÇÃO”, "SENTIMENTO", 
“ALUNO", "PROFESSOR” e "EDUCAÇÃO”, com suas variações no plural, e variações na língua inglesa. Após a localização dos termos, líamos o parágrafo visando entender se estava contextualizado com a pesquisa. Como resultado da segunda etapa, o número de Teses foi reduzido para 11 e o de Dissertações para 59.

Posteriormente, foram lidos título, resumo e conclusão de cada trabalho com o objetivo de identificar se os documentos coletados efetivamente se referiam ao uso da Computação Afetiva, e se o uso deste estava relacionado a algum processo ensino-aprendizagem. Como resultado da última etapa da busca chegamos às 08 Teses e 13 Dissertações, totalizando 21 trabalhos. A relação das Teses e Dissertação resultantes desta busca encontram-se disponíveis em https://bit.ly/2FVf94R.

\subsection{Pré processamento}

Na criação da base de dados para as redes de citação, inicialmente foram extraídos das 21 Teses e Dissertações alvo dessa pesquisa todos os trabalhos citados, e foi gerado um documento único. Posteriormente, como segunda etapa do pré-processamento, foram identificados: o nome dos trabalhos citados, seus respectivos autores, e o ano da publicação, como sendo os dados comuns a todos os trabalhos citados.

$\mathrm{Na}$ terceira etapa do processo de pré-processamento criamos um segundo documento com os dados identificados na etapa anterior, e posteriormente aplicamos a esses dados um padrão único de escrita visando otimizar o processamento. Para os trabalhos citados, adotamos como padrão, a conversão dos caracteres para caixa-alta, a supressão de caracteres especiais, acentos e espaços. No caso do nome dos autores, adotamos como padrão o sobrenome finalizado por vírgula(,) seguido pela inicial dos demais nomes do autor finalizados por ponto(.), todos em caixa-alta, e caso o trabalho citado possuísse mais de um autor, os nomes foram separados por ponto e vírgula (;) ao final de cada um. Por fim, nos trabalhos citados, que possuíam uma letra ao final do ano de publicação, essa foi suprimida.

Após o pré-processamento da base de dados foram construídas as redes de citação, e utilizadas para a obtenção dos resultados.

\subsection{Redes de citação}

As citações indicam de quais fontes um autor fez uso para conceber e fundamentar suas ideias, logo, a análise das citações em uma determinada área demonstra quais são os trabalhos influenciadores, e seus devidos autores de referência [MORAES, FURTADO \& TOMAÉL(2015)].

Para analisar os dados das citações das Teses e Dissertações foram construídas duas redes de citações: a primeira rede trata o pesquisador de cada tese/dissertação, ou autor referência como um nó, e a relação entre eles como arestas considerando, que cada citação tenha pelo menos um autor referência, indicando a relação entre os mesmos. Já na segunda rede de citação, consideramos como nós os pesquisadores das teses/dissertações, e os trabalhos citados, mantendo a relação deles como arestas. As redes de citações têm como objetivo mostrar as relações entre todos os nós, e evidenciar as suas estruturas.

\section{Resultados}

Após o pré-processamento, inicialmente, foram analisados os dados primários de 21 pesquisas stricto-sensu focadas em Computação Afetiva no ambiente computacional. 


\subsection{Dados primários}

Ao analisarmos as pesquisas por instituição de ensino observamos que 04 eram publicações da Pontifícia Universidade Católica do Paraná (PUC-PR), 04 da Universidade de São Paulo (USP-SP), 02 da Universidade Federal de Uberlândia, e que os demais trabalhos estavam distribuídos entre Universidades Federais: de Alagoas, do Paraná, de Pernambuco, do Rio Grande do Norte, do Rio Grande do Sul, do Rio de Janeiro, de Santa Maria(RS) e Sergipe, além da Pontifícia Universidade Católica de Minas Gerais, Universidade Estadual do Ceará e Universidade Cândido Mendes, com isso entendemos que embora haja pequena concentração nas duas primeiras instituições citadas, não há uma indicação de que existam, nessas instituições, uma pesquisa continuada sobre o uso da Computação Afetiva e seus impactos na educação brasileira. A Figura 1 apresenta a distribuição destas publicações pelos Estados das instituições de ensino.

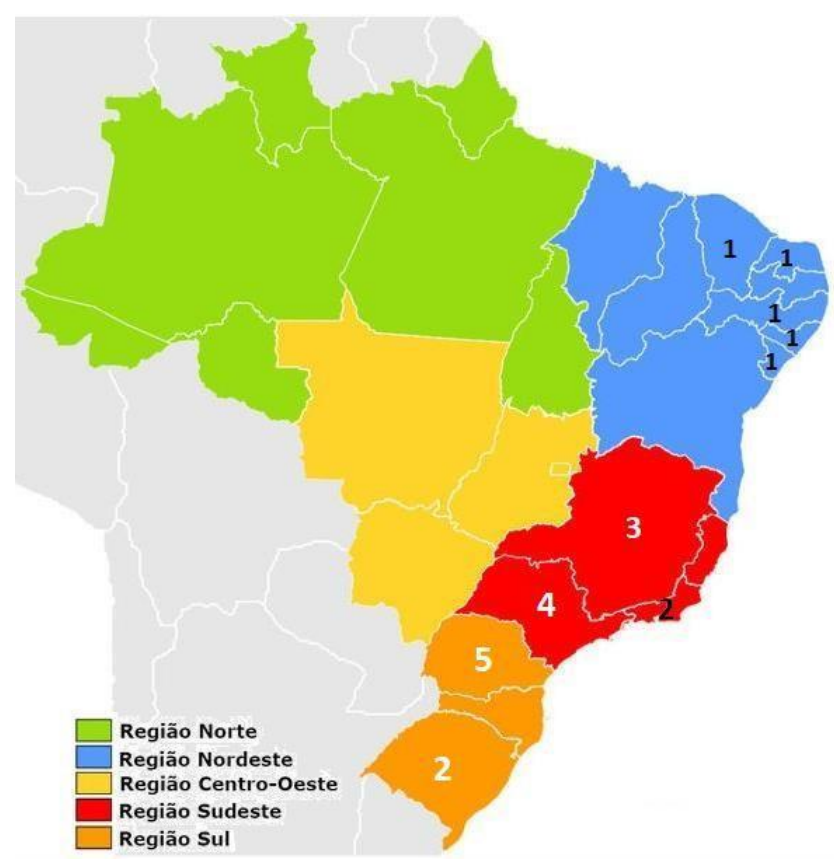

Figura 1 - Distribuição de Teses e Dissertações sobre Computação Afetiva por estado (2013-2018)

Ao considerarmos as pesquisas por ano de defesa, observamos que entre 2013 e 2015, conforme pode ser observado na Figura 2, houve apenas 05 defesas ligadas ao tema de Computação Afetiva. Nos anos de 2016 e 2017, houve um crescimento das pesquisas, sendo de 05 defesas em 2016, e 09 em 2017, porém no ano de 2018 o número de defesas sobre Computação Afetiva voltou ao mesmo patamar do início da série. Observamos que o aumento das pesquisas estava vinculado, principalmente, a associação do uso da Computação Afetiva com a educação a distância, e jogos sérios. 
IX Congresso Brasileiro de Informática na Educação (CBIE 2020)

Anais do XXXI Simpósio Brasileiro de Informática na Educação (SBIE 2020)

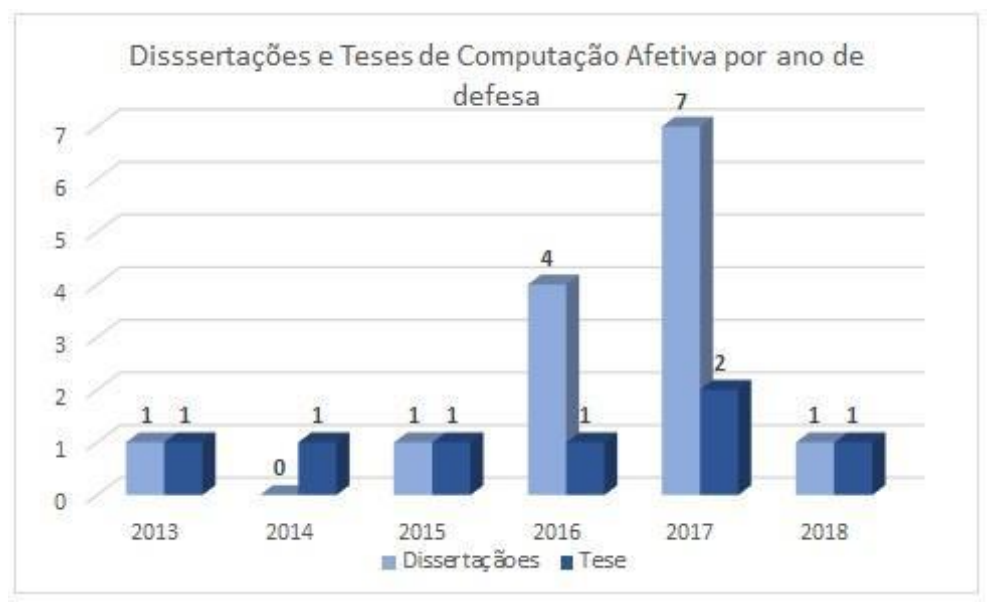

Figura 2 - Distribuição de Teses e Dissertações sobre Computação Afetiva por ano de defesa

$\mathrm{Na}$ análise, não consideramos o ano de 2019, uma vez que, no momento da escrita deste trabalho, ao realizarmos a pesquisa no CTD, o mesmo não informava nenhum trabalho dentro dos critérios de busca.

Por fim, em relação aos dados primários, analisamos a quantidade de trabalhos citados em cada uma das pesquisas. Sendo o total de trabalhos citados de 2865 nas 21 Teses e Dissertações.

Segundo a ABNT, as citações são "a menção da informação extraída de outra fonte" e segundo MORAES, FURTADO \& TOMAÉL(2015) as redes de citação são consideradas uma rede social da formação do saber das pesquisas e, nesse sentido, criamos uma classificação das pesquisas baseado na mediana geral do número de trabalhos e a mediana dos trabalhos citados por tipo de pesquisa stricto-sensu. Essa classificação é apresentada nas Figuras 3 e 4.

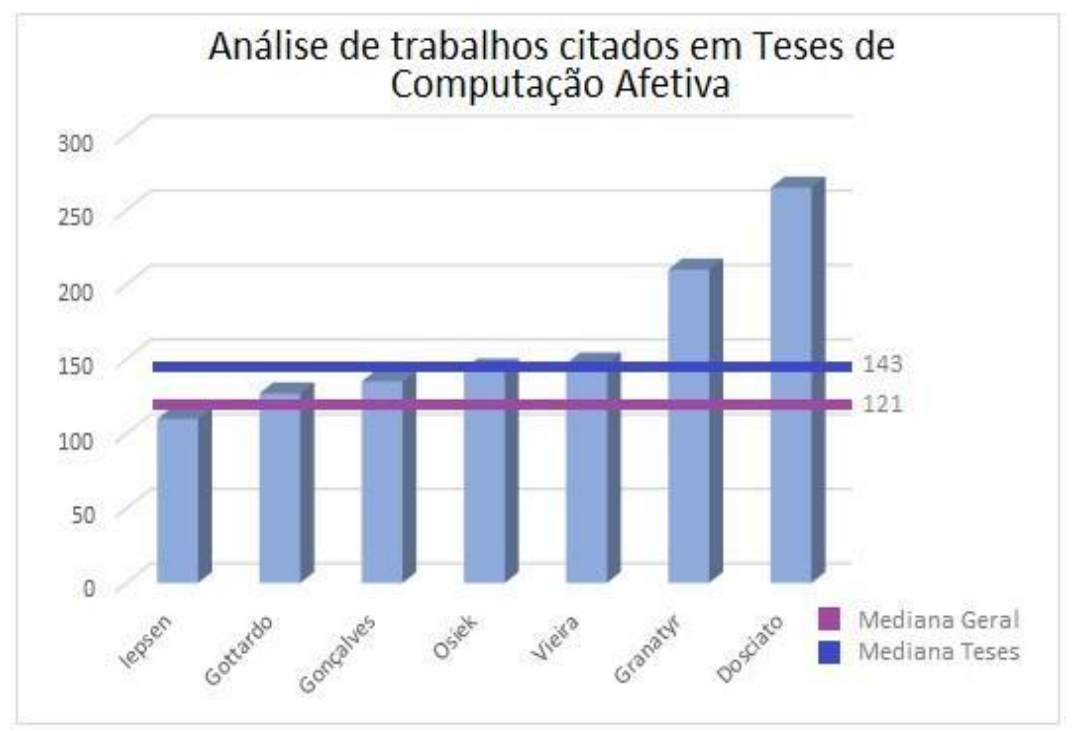

Figura 3 - Número de trabalhos citados em dissertações de Computação Afetiva (2013-2018) 


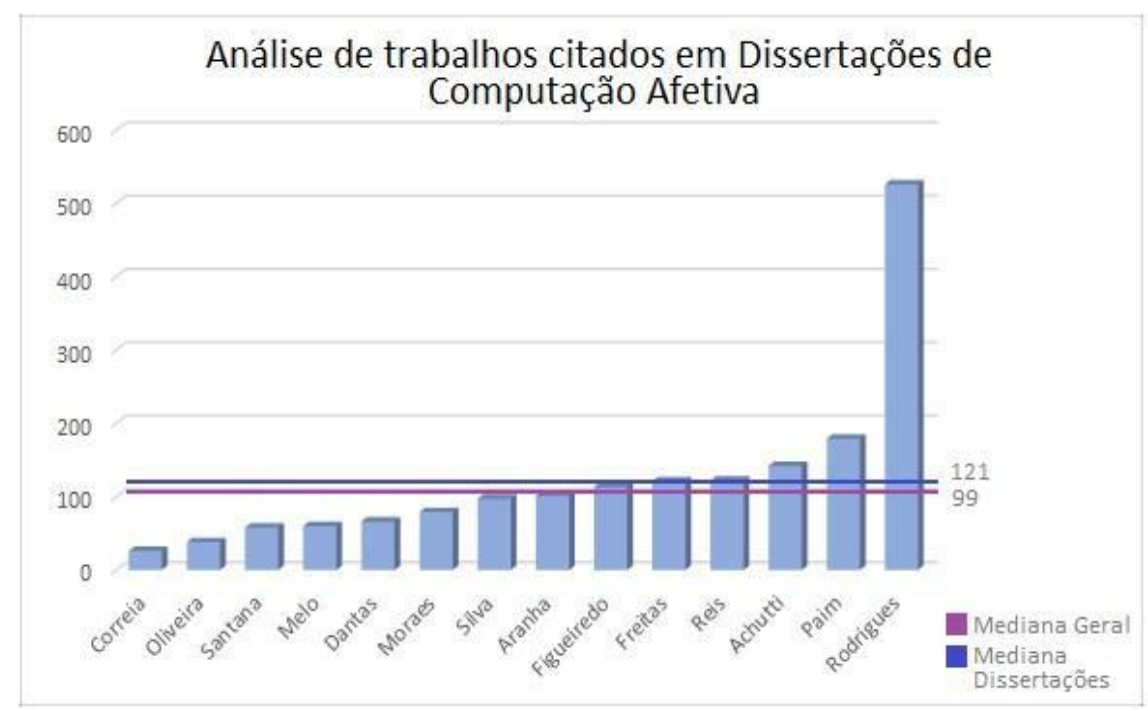

Figura 4 - Número de trabalhos citados em teses de Computação Afetiva (2013-2018)

Ao analisarmos o número de trabalhos citados, destacamos como sendo as pesquisas com maior número de citações, a dissertação P18 com 526 trabalhos citados e a Tese P05 com 265 trabalhos citados, e com menor número de citações a dissertação P03 com apenas 40 trabalhos citados.

\subsection{Redes de citação}

Após a execução do pré-processamento das pesquisas stricto-sensu, foram obtidos os 2865 trabalhos, sendo que nestes foram citados 1225 autores e coautores (autores referência). Esses trabalhos geraram a base de dados utilizada para a criação às duas redes de citação, analisadas neste trabalho.

\subsubsection{Redes de citações - Teses〈Dissertações - Autores Referência}

Como resultado do processamento para criação das redes de citações, TeselDissertações - Autores Referência identificamos, que a pesquisa com mais autores referência citados, utilizou 1225 autores referência enquanto a pesquisa, que utilizou menos, citou apenas 40 autores referência, distintos.

Na Figura 5 é possível observar, que a rede Teses\Dissertação - Autores Referência, possui apenas 01 componente conexo, indicando que todos os pesquisadores utilizaram ao menos 01 autor de referência comum às demais pesquisas.

É importante considerarmos que um mesmo autor de referência pode ter sido autor, ou coautor, em diversos trabalhos citados, e que um único trabalho citado pode possuir mais de um autor de referência, CUNNINGHAN et al. apud OSIEK(2014), por exemplo, destaca-se por possuir 16 autores. 


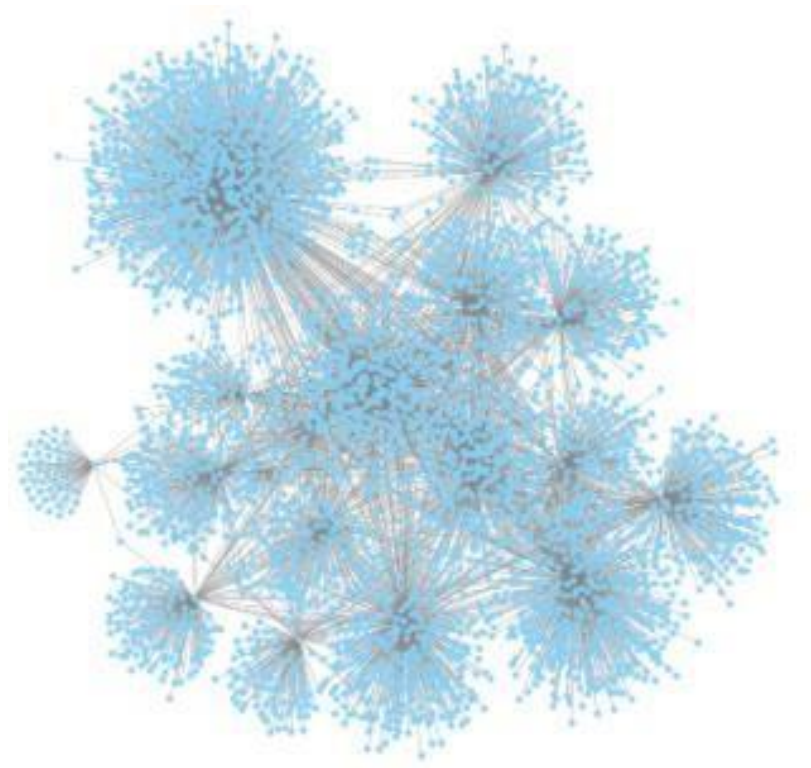

Figura 05 - Rede de citação Teses-Dissertações - Autores referência

Ao analisarmos os resultados obtidos com o processamento da primeira rede é possível afirmar que o autor de referência mais citado ao pesquisarmos sobre Computação Afetiva na área educacional é PICARD, R.W. sendo citada em 17 de 21 pesquisas analisadas, mas destacam-se ainda, EKMAN, P., com 15 citações, NUNES, M.A.S.N., com 14 citações e JAQUES, P.A. com 11 citações.

Após identificar quem são os autores mais citados entre as pesquisas stricto-sensu analisadas, observamos que estes autores são mais comumente utilizados para: PICARD definição da Computação Afetiva, a associação da Computação Afetiva com ambientes educacionais e correlacionando Computação Afetiva com IHC, já as citações relacionadas a EKMAN estão associadas diretamente a definição de emoções básicas enquanto as citações das autoras NUNES e JAQUES estão associadas principalmente a Computação Afetiva na educação, e ao uso de pesquisas e tecnologias afetivas na educação brasileira.

Também foi possível observar que as autoras PICARD, NUNES e JAQUES vão além da influência direta com obras de autoria exclusiva, uma vez que todas possuem influência direta de diversos trabalhos como coautoras e indiretas com citações em outros trabalhos citados neste estudo.

\subsubsection{Redes de citações - Teses $\backslash$ Dissertações - Trabalhos Citados}

Como resultado do processamento do algoritmo para criação de rede de citações TeselDissertações - Trabalhos Citados, foi possível identificar quais e quantos foram os trabalhos citados por cada um dos pesquisadores sendo que o pesquisador que mais citou trabalhos em sua pesquisa utilizou 526 trabalhos, o pesquisador com menor número de trabalhos citados, o fez com 26 trabalhos.

É importante ressaltar, que um mesmo pesquisador, pode citar um mesmo trabalho em vários momentos de sua pesquisa, porém nossa rede considerou não a quantidade de vezes que o mesmo trabalho foi citado na mesma pesquisa, mas a quantidade de vezes que o trabalho foi citado em pesquisas diferentes. 
Observando a rede Teses\Dissertações - Trabalhos Citados, na Figura 6, é possível visualizar 2 componentes conexos, indicando que um autor utilizou trabalhos exclusivos, porém todos os outros 20 pesquisadores compartilharam pelo menos 1 trabalho comum com os outros pesquisadores.

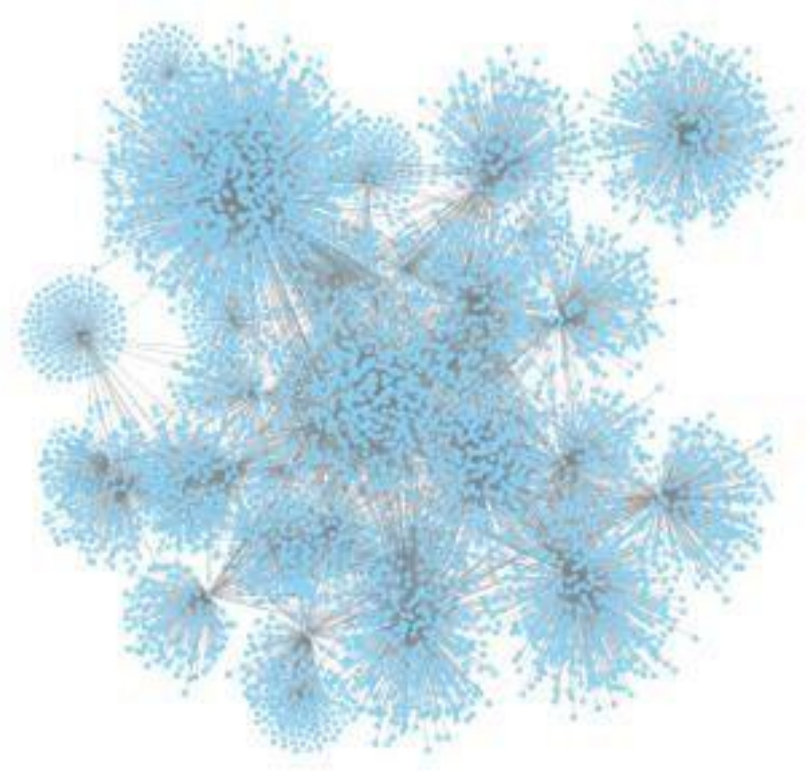

Figura 06 - Rede de citação Teses-Dissertações - Trabalhos referência

O trabalho que mais obteve citações foi Affective Computing, de PICARD, R.W., sendo citado em 11 de 21 pesquisas, em seguida The Cognitive Structure of Emotions, de ORTONY, A., sendo citado em 08 de 21 pesquisas.

Ao analisarmos a rede de citações TeselDissertações - Trabalhos Citados foi possível avaliar o ano de publicação dos trabalhos citados. A Figura 7 mostra a distribuição do ano de publicação dos trabalhos citados, considerado o período de 1980 a 2018.

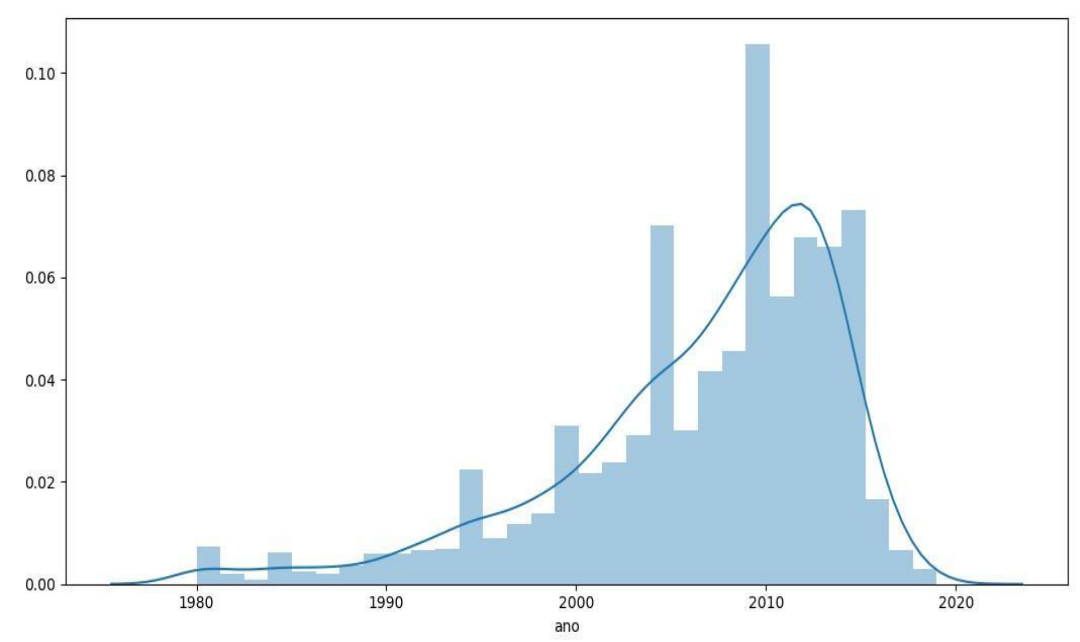

Figura 07 - Distribuição de trabalhos citados por ano de publicação 
IX Congresso Brasileiro de Informática na Educação (CBIE 2020)

Anais do XXXI Simpósio Brasileiro de Informática na Educação (SBIE 2020)

\section{Considerações Finais}

Este artigo apresentou uma análise bibliométrica focada em criar e analisar as redes de citações sobre Computação Afetiva em ambientes computacionais de ensinoaprendizagem no Brasil. Para tal, foram analisadas as referências bibliográficas de 21 Teses e Dissertações. Como parte de nossa metodologia estabelecemos 03 objetivos como bússolas orientadoras desta pesquisa. (i) Identificar quantos e quais foram as pesquisas stricto-sensu sobre Computação Afetiva em ambientes computacionais entre 2013 e 2018. (ii) Identificar quantos, e quais são os trabalhos mais citados nessas pesquisas. (iii) Identificar quais são os autores com maior influência nessas pesquisas.

Em relação ao primeiro objetivo (i), foram identificadas 21 pesquisas stricto-sensu sobre Computação Afetiva, sendo 08 Teses e 13 Dissertações. Ainda, foi possível estabelecer que as instituições de ensino que mais realizaram pesquisas foram a PUC-PR e a USP$\mathrm{SP}$, com 4 pesquisas cada, e que a maior quantidade de defesas sobre o tema aconteceu em 2017.

Sobre o segundo objetivo (ii), desenvolvemos uma rede de citações com 2865 trabalhos citados, e ao analisá-los foi possível observar que apenas 01 dos pesquisadores utilizou referências que não foram citadas em outros trabalhos, o que indica que os demais autores utilizaram uma rede de trabalhos comuns em suas pesquisas, mostrando com isso, que embora com abordagens diferentes, os pesquisadores foram influenciados por uma mesma rede de trabalhos sobre o tema.

Em relação aos autores (iii) identificamos, que a autora PICARD, R.W. é a mais citada entre os trabalhos pesquisados, sendo referenciada em 16 de 21 trabalhos pesquisados, e que outros como EKMAN, NUNES, JAQUES também são frequentemente utilizados nas pesquisas sobre Computação Afetiva na área da educação. Observamos, também, que autores como PICARD, NUNES e JAQUES, possuem muitas publicações sobre o tema, não apenas como autores principais, mas também como coautores.

Ao analisarmos os resultados alcançados é possível concluir que as pesquisas sobre o uso da Computação Afetiva no ambiente computacional de ensino-aprendizagem no Brasil possuem uma rede de citações comum, e que esta rede é alimentada por autores de grande relevância nas publicações nacionais.

Como trabalhos futuros pretendemos analisar com maior profundidade os objetivos das pesquisas stricto-sensu utilizadas neste artigo, suas metodologias, contribuições e limitações de pesquisa.

\section{Referências Bibliográficas}

FULLER, R. M., VICIAN, C. M., BROWN, S. A., Longitudinal Effects of Computer Mediated Communication Anxiety on Interaction in Virtual Teams. Research Online Accounting Faculty Publications Accounting, University of St. Thomas, Minnesota UST. 2016.

GONÇALVES, V.P., Uma abordagem para indicar o estado emocional de usuários em tempo de interação. Programa de Pós-Graduação da Universidade de São Paulo. 2016.

GONZALEZ, R. C., WOODS, R. E., Computação Afetiva personalizando interfaces, interações e recomendações de produtos, serviços e pessoas em ambientes 
computacionais. Projetos e Pesquisas em Ciência da Computação no DCOMP/PROCC/ UFS. 2012.

GOTTARDO, E., Inferência de estados afetivos em ambientes educacionais - proposta de um modelo híbrido baseado em informações cognitivas e físicas. Programa de PósGraduação da Universidade Federal do Paraná. 2018.

HORTON JR., F. W.; Understanding information literacy: a primer. Paris: UNESCO, 2008.

MORAES, M., FURTADO, R.L., TOMAÉL, M.I., Redes de Citação: estudo de redes de pesquisadores a partir da competência em informação. Revista Em Questão. Porto Alegre, v. 21, n. 2, p. 181-202. 2015.

NIJHOLT, A., POPPE, R., Facial and Bodily Expressions for Control and Adaptation of

Games. ECAG 2008 Workshop. Centre for Telematics and Information Technology (CTIT). 2008.

OSIEK, B.A.; Reconhecimento de sentimento em texto abordado através da computação afetiva. Programa de Pós-graduação em Engenharia de Sistemas e Computação da Universidade Federal do Rio de Janeiro, 2014.

PAIM, A.M., Inferência de personalidade a partir de textos em português brasileiro utilizando léxicos. Programa de Pós-Graduação da Pontifícia Universidade Católica do Paraná. 2016.

PICARD, R.W., Affective computing. Cambridge: MIT Press, 1997.

SANTANA, A.O., Geração de Ambientes Virtuais de Ensino-Aprendizagem para Cursos Baseados na Realização de Projetos. Programa de Pós-Graduação da Universidade Federal do Rio Grande do Norte. 2017.

STIPEK, D. J., Motivation to learn: Integrating theory and practice. Education Technology and Computer Science (ETCS) International Workshop on. 2002.

TAN, C. T., ROSSER, D., BAKKES, S. E. R., PISAN, Y., A feasibility study in using facial expressions analysis to evaluate player experiences. Proceedings of The 8th Australasian Conference on Interactive Entertainment Playing the System - IE '12. 2012.

ZUO, X., FENG, B., YAO, Y., ZHANG, T., ZHANG, Q., WANG, M., ZUO, W.,

Correlation-based Feature Subset Selection for Machine Learning. 1998. 